\title{
Control Point Position Calibration for Light Pen Coordinate Measuring System
}

\author{
Sen Wang ${ }^{1 *}$, Shugui Liu ${ }^{1}$, and Qing Mao ${ }^{1}$ \\ ${ }^{1}$ State Key Laboratory of Precision Measuring Technology and Instruments, Tianjin University, 300072 Tianjin, China
}

\begin{abstract}
Light pen coordinate measuring system is a kind of vision-based portable coordinate measuring technique. It implements coordinate measuring by analyzing the image of light pen, which has several control points. With the relative positons of the control points determined and the pixel coordinates of their image points acquired by image processing, the posture of light pen can be obtained by solving perspectiven-point problem. Therefore, the control point positons serve as the measuring basis of the whole system. However, the accuracy of existing calibration methods is not satisfactory. In this paper, a new method of control point positon calibration, which utilizes traditional coordinate measuring machine, is proposed. The light pen is fasten to the measuring arm of a coordinate measuring machine and moves along a three dimensional grid route driven by the machine. A stationary camera is located in front of the machine to capture the image of light pen at each node of the route. For each control point, an individual coordinate system is established. Then the relative positions of control points can be transformed into the differences of a series of translation vectors. Experiments show that, with the proposed method, the accuracy of light pen coordinate measuring system can reach $30 \mu m$ within 10 meters.
\end{abstract}

\section{Introduction}

Coordinate measuring is the key technique in manufacturing industry and reverse engineering [1]. To meet the increasing demand of in-situ measurement of large work pieces, several kinds of portable coordinate measuring system arise in the last two decades. Among them, light pen coordinate system (LPCMS) is indispensable for its ability to measure invisible part of work pieces $[2,3]$.

A typical LPCMS is composed of a camera, a laptop, a light pen and some accessories. The light pen has several control points and a contact probe on it. Generally, the control points are active luminous infrared light emitting diodes (LED). Once they are installed on the light pen, their relative positons are fixed. LPCMS implements coordinate measuring by analysing the image of light pen captured by a stationary camera. With the relative positions of control points and the pixel coordinates of their image points, the posture of light pen can be calculated by solving perspective-n-point (PnP) problem, which is well known in the domain of computer vision. Therefore, the relative positions of control points serve as the measuring basis of LPCMS. The measurement accuracy of the whole system is highly dependent on the accuracy of control point positon calibration. How to calibrate the relative positons of the control points is the most crucial issue in system calibration.

There exist two kinds of methods to calibrate the control point positions. One kind is to measure the control point positions directly utilizing image measuring apparatus together with traditional coordinate measuring machines (CMM) [4]. However, the LED has a certain size, and therefore cannot be treated as an ideal point. What the calibration really need to determine is the relative positions of the luminous centres of the control points, which cannot be directly and accurately located. Therefore, this kind of method can only obtain a rough result. The other kind is the structure from motion (SFM) method in computer vision [5]. Because it is a vision-based method, it naturally calculates the luminous centres of the control points, and therefore can overcome the disadvantage of the direct measurement method mentioned above. It uses a moving camera to capture a group of images of light pen from several different viewpoints. The relative positons of control points can be recovered up to a scale from this group of images using bundle adjustment method. Then the absolute dimension can be obtained by resorting to a calibration bar, which has accurate lengths. The problem with this kind of method is that, the calibration accuracy is strongly affected by the calibration accuracy of the camera. The solving complexity of bundle adjustment limits the number of different viewpoints adopted by the calibration procedure. What's more, using the lengths on the calibration bar as measuring basis will also make the calibration result unreliable.

In this paper, a new calibration method of control point positions based on CMM is proposed. It utilizes tens of thousands accurate translations generated by $\mathrm{CMM}$ as measuring basis. The light pen is fasten to the

\footnotetext{
* Corresponding author: senwang@tju.edu.cn
} 
measuring arm of a CMM and moves along a three dimensional grid driven by the CMM. A stationary camera is located in front of the CMM to capture the image of the light pen at each node of the route. At the same time, the readings of CMM are also recorded. For each control point, an individual coordinate system is established according to the following condition. At each node of the route, the coordinate of a control point in its own coordinate system is equal to the corresponding reading of the CMM. With the readings of CMM and the pixel coordinates of the control points, the translation between all the control point coordinate systems and the camera coordinate system can be calculated. After a simple conversion, the relative positons of the control points can be recovered from the differences of these translation vectors. Compared with the first kind of previous method, the proposed method is also a visionbased method, which can avoid the problem of direct measurement. Compared with the second kind of previous method, the accuracy of the proposed method is guaranteed by the accuracy of CMM, which is very high. The establishment of the control point coordinate systems makes the solving process not complicated and able to handle tens of thousands of nodes on the CMM route, so that the influence of the random errors can be reduced to a very low level. Therefore, the proposed method is superior to the previous methods.

Section 2 introduces the mathematical model of LPCMS and the proposed calibration method of control point positions. Section 3 gives the results of the calibration experiment and the measurement experiment of the whole LPCMS. Then a brief discussion of the performance of the proposed method is also given. Section 4 concludes the paper.

\section{Control Point Positon Calibration}

As shown in Fig. 1, a typical LPCMS is composed of a camera, a laptop, a light pen and some accessories.

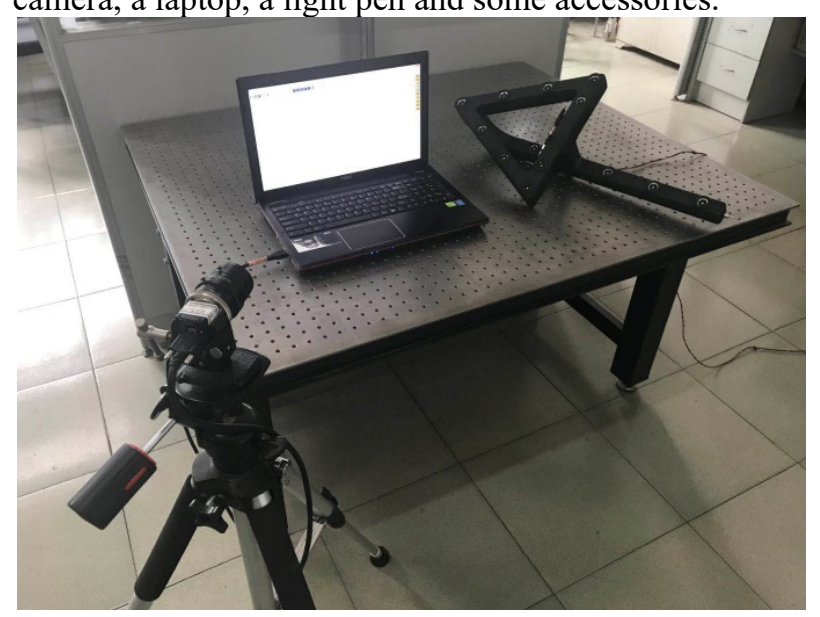

Fig. 1. Typical composition of LPCMS.

Fig. 2 gives the light pen structure adopted in this paper. It has 13 control points. The fifth to thirteenth control points are distributed in the front plane and form an isosceles triangle. The first to fourth control points are distributed in a line that is parallel to the front plane. The mian task of this paper is to determine the relative positions of these 13 control points.

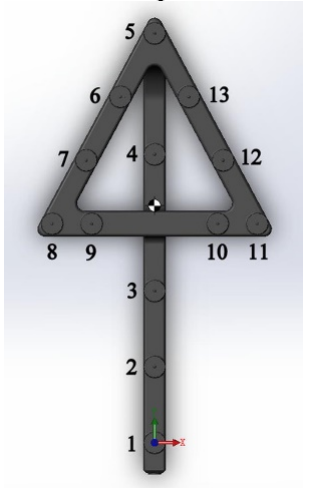

Fig. 2. Distribution of control points in this paper.

\subsection{Mathematical Model of LPCMS}

For the convenience of description, the camera coordinate system is denoted as $O_{c}-x_{c} y_{c} z_{c}$ and the light pen coordinate system is denoted as $O_{l}-x_{l} y_{l} z_{l}$. Because the position of the camera is fixed during measurement, $O_{c}-x_{c} y_{c} z_{c}$ can be regarded as the reference system of LPCMS. For any space point $\mathbf{P}$, its coordinate in $O_{c}-x_{c} y_{c} z_{c}$ is denoted as $\mathbf{P}_{c}=\left(x_{c}, y_{c}, z_{c}\right)^{\mathbf{T}}$ and its homogenous coordinate in $O_{l}-x_{l} y_{l} z_{l}$ is denoted as $\tilde{\mathbf{P}}_{l}=\left(x_{c}, y_{c}, z_{c}, 1\right)^{\mathbf{T}}$. These two coordinates satisfies the three dimensional rigid body transformation:

$$
\mathbf{P}_{c}=[\mathbf{R} \mid \mathbf{T}] \tilde{\mathbf{P}}_{l}
$$

where $\mathbf{R}$ and $\mathbf{T}$ are the rotation matrix and translation vector between $O_{c}-x_{c} y_{c} z_{c}$ and $O_{l}-x_{l} y_{l} z_{l}$ respectively. The homogenous pixel coordinate of the image of point $\mathbf{P}$ is denoted as $\tilde{\mathbf{p}}=(u, v, 1)^{\mathbf{T}}$, then according to the imaging model of the camera, the following equation is obtained:

$$
\lambda \tilde{\mathbf{p}}=\mathbf{K} \mathbf{P}_{c}=\mathbf{K}[\mathbf{R} \mid \mathbf{T}] \tilde{\mathbf{P}}_{l}
$$

where $\mathbf{K}$ is the intrinsic parameter matrix of the camera, which can be determined by camera calibration $[6,7]$, and $\lambda$ is a nonzero scale factor.

If the relative positons of the control points are calibrated, then their coordinates in $O_{l}-x_{l} y_{l} z_{l}$ are determined. The pixel coordinates of the image of control points can be obtained by image processing. Then, for each control point, two equations can be derived form (2) by eliminating scale factor $\lambda$. If the number of control points is sufficient, an overdetermined nonlinear equation system is established. The posture of light pen, which is represented by rotation matrix $\mathbf{R}$ and translation vector $\mathbf{T}$, can be determined by solving this equation system using nonlinear least squares method. This process is well known as PnP problem in the field of computer vision. With the posture of the light pen 
determined, the coordinate of the probe tip centre in $O_{l}-x_{l} y_{l} z_{l}$, which is calibrated in advance [8], can be transformed to the coordinate in $O_{c}-x_{c} y_{c} z_{c}$ according to (1). The coordinate of the measured point in $O_{c}-x_{c} y_{c} z_{c}$, which is exactly the final measuring result, can be acquired after a simple radius compensation.

\subsection{Calibration Method}

In this paper, a new method of control point positon calibration, which utilizes CMM, is proposed. The light pen is fasten to the measuring arm of a CMM and moves along a preprogramed three dimensional grid route driven by the CMM. A stationary camera is placed in front of the CMM to capture the image of the light pen at each node of the route. At the same time, the readings of the CMM are also recorded. Suppose there are ${ }^{n}$ control points on the light pen. For each control point, an individual coordinate system is established, so that the coordinate of a control point in its own coordinate system is equal to the corresponding reading of the CMM.

As shown in Fig. 3, the machine coordinate system of the CMM is denoted as $O_{m}-x_{m} y_{m} z_{m}$, and its origin is denoted as $\mathbf{O}_{m}$. The control point coordinate systems are denoted as $O_{i}-x_{i} y_{i} z_{i},(i=1,2, \cdots, n)$, and their origins are denoted as $\mathbf{O}_{i},(i=1,2, \cdots, n)$. The probe tip centre of the CMM is denoted as point $\mathbf{M}$, and the luminous centres of the control points are denotes as $\mathbf{L}_{i},(i=1,2, \cdots, n)$. The reading of the CMM is exactly the coordinate of point $\mathbf{M}$ in $O_{m}-x_{m} y_{m} z_{m}$. Then the establishment condition of $O_{i}-x_{i} y_{i} z_{i},(i=1,2, \cdots, n)$ can be formulated as:

$$
\overrightarrow{\mathbf{O}_{m} \mathbf{M}}=\overrightarrow{\mathbf{O}_{i} \mathbf{L}_{i}},(i=1,2, \cdots, n)
$$

The four points

$$
\mathbf{O}_{m}, \mathbf{M}, \mathbf{O}_{i}, \mathbf{L}_{i},(i=1,2, \cdots, n)
$$

always form a parallelogram at each node of the CMM route. Take the first two control points for example:

$$
\begin{gathered}
\overrightarrow{\mathbf{M L}_{1}}=\overrightarrow{\mathbf{O}_{m} \mathbf{O}_{1}}, \overrightarrow{\mathbf{M L}_{2}}=\overrightarrow{\mathbf{O}_{m} \mathbf{O}_{2}} \\
\overrightarrow{\mathbf{L}_{1} \mathbf{L}_{2}}=\overrightarrow{\mathbf{M L}_{2}}-\overrightarrow{\mathbf{M L}_{1}}=\overrightarrow{\mathbf{O}_{m} \mathbf{O}_{2}}-\overrightarrow{\mathbf{O}_{m} \mathbf{O}_{1}}=\overrightarrow{\mathbf{O}_{1} \mathbf{O}_{2}}
\end{gathered}
$$

From (4), it can be seen that the relative positions of $\mathbf{L}_{i},(i=1,2, \cdots, n)$ is the same as the relative positions of $\mathbf{O}_{i},(i=1,2, \cdots, n)$

For all the control point coordinate systems, their translation vectors relative to camera coordinate system, which can be denoted as $\mathbf{T}_{i},(i=1,2, \cdots, n)$, can be obtained by solving PnP problem just like what is mentioned above. After that, the relative positons of $\mathbf{O}_{i},(i=1,2, \cdots, n)$ can be calculated form the differences of these translation vectors. Take the first two control points for example:

$$
\overrightarrow{\mathbf{O}_{1} \mathbf{O}_{2}}=\mathbf{T}_{2}-\mathbf{T}_{1}
$$

After these two steps of conversion, the problem of control point position calibration is converted to solving a series of PnP problems. The solving process is not complicated, and therefore the calibration procedure allows that there are tens of thousands of nodes on the CMM route.

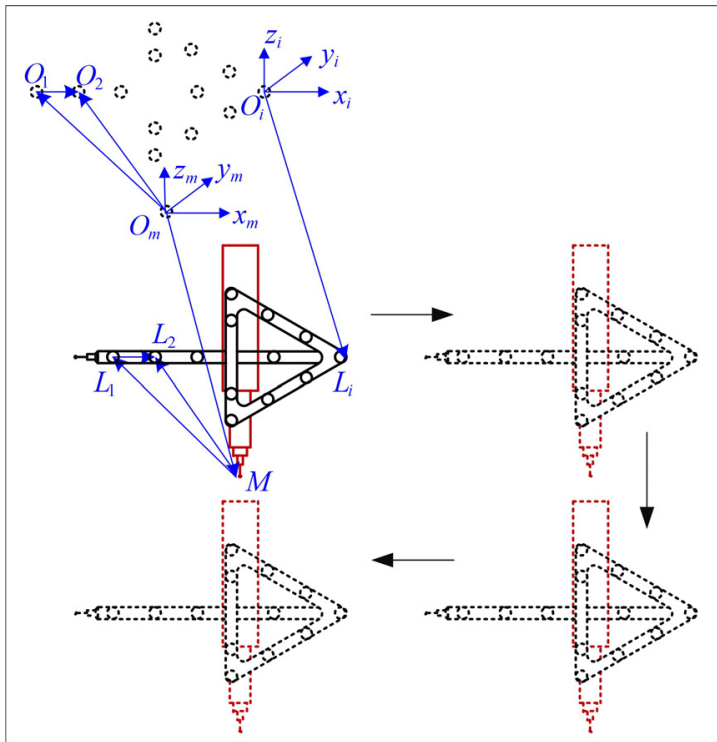

Fig. 3. The establishment of control point coordinate system.

\subsection{Light pen coordinate system establishment}

From the calibration procedure above, the relative positions of the control points can be obtained from the differences of a series of translation vectors. So far, the control point positons are represented with coordinates in the camera coordinate system, which vary with the position of the camera. This will result in that the results of two calibration experiments for the same light pen may look totally different. For ease of use, the light pen coordinate system is established according to the structure of the light pen, and the control points positions are converted to be represented by coordinates in light pen coordinate system after a three dimensional rigid body transformation. Take the light pen in Fig. 2 for example, the luminous centre of the first control point is regarded as the origin. The normal vector of the front plane is regarded as the z-axis, and the direction vector of the back line is regarded as the y-axis. Then, $x$-axis can be obtained from the cross product of $y$-axis and $z$ axis. By this means, the calibration result will reflect the structure more intuitively.

\section{Experiments and Discussion}

Fig. 4 gives the scene of one calibration experiment. The light pen is fasten to the measuring arm of the CMM using a specially customized fixture. There are $20 \times 20 \times 20=8000$ nodes on the three dimensional grid route of the CMM. The calibration can be finished within 3 hours. 
With the proposed method, the coordinates of the control points in light pen coordinate system are listed in Table 1.

With the calibration results of control point positons in Table 1, LPCMS is used to measure three standard gauges, whose lengths are $100 \mathrm{~mm}, 250 \mathrm{~mm}$, $1000 \mathrm{~mm}$ respectively, at different distances from 2 to 10 meters. Owing to space constraints, only the statistical measurement results are shown in Table 2.

From the results in Table 2, it can be seen that, with the proposed method, the accuracy of LPCMS cam reach $20 \mu m$ within 10 meters, and the standard deviation of measurement of LPCMS can reach $30 \mu m$ within 10 meters. These results demonstrate that the proposed method of control point position calibration is superior to the previous methods, and therefore can improve the accuracy of the whole LPCMS. The only disadvantage of the proposed method is that it takes several hours to finish one calibration.

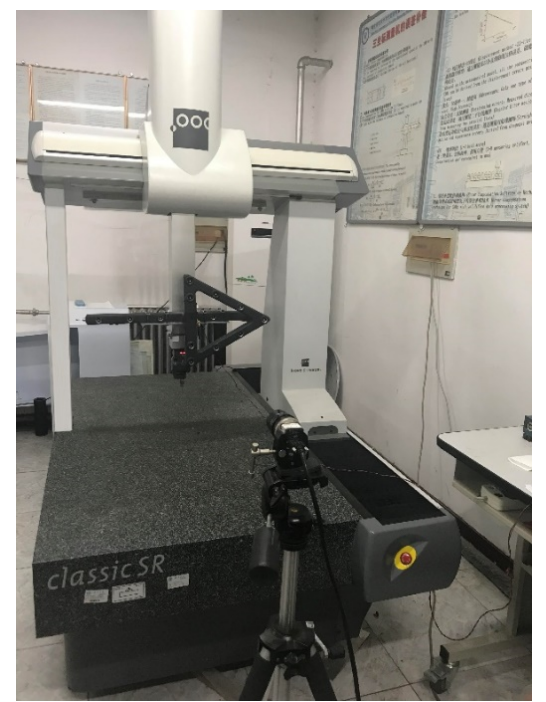

Fig. 4. Scene of calibration experiment.

Table 1. Coordinates of control points in light pen coordinate system. (mm)

\begin{tabular}{|c|c|c|c|}
\hline$i$ & $x_{i}$ & $y_{i}$ & $z_{i}$ \\
\hline 1 & 0.000 & 0.000 & 0.000 \\
\hline 2 & -0.228 & 99.159 & -0.064 \\
\hline 3 & -0.025 & 199.053 & -0.071 \\
\hline 4 & 0.730 & 379.013 & -0.024 \\
\hline 5 & -0.874 & 538.094 & 100.333 \\
\hline 6 & -45.681 & 454.440 & 100.167 \\
\hline 7 & -90.222 & 371.082 & 100.043 \\
\hline 8 & -134.657 & 287.086 & 99.951 \\
\hline 9 & -79.488 & 287.447 & 100.223 \\
\hline 10 & 79.949 & 288.113 & 99.950 \\
\hline 11 & 134.962 & 287.970 & 100.048 \\
\hline 12 & 89.451 & 371.514 & 100.119 \\
\hline 13 & 43.858 & 455.096 & 100.176 \\
\hline
\end{tabular}

Table 2. Statistical measurement results of LPCMS. (mm)

\begin{tabular}{|c|c|c|c|c|}
\hline \multicolumn{2}{|c|}{ Distance } & $2 \mathrm{~m}$ & $6 \mathrm{~m}$ & $10 \mathrm{~m}$ \\
\hline \multirow{2}{*}{$\begin{array}{l}\text { 100mm } \\
\text { gauge }\end{array}$} & Ave & 99.994 & 100.006 & 99.992 \\
\hline & Std & 0.0025 & 0.0048 & 0.0071 \\
\hline \multirow{2}{*}{$\begin{array}{c}\mathbf{2 5 0 m m} \\
\text { gauge }\end{array}$} & Ave & 250.009 & 249.983 & 250.007 \\
\hline & Std & 0.0028 & 0.0072 & 0.0158 \\
\hline \multirow{2}{*}{$\begin{array}{l}1000 \mathrm{~mm} \\
\text { gauge }\end{array}$} & Ave & 999.982 & 1000.015 & 999.979 \\
\hline & Std & 0.0062 & 0.0187 & 0.0315 \\
\hline
\end{tabular}

\section{Conclusion}

In this paper, a new CMM-based method of control point position calibration for LPCMS is proposed. It utilizes accurate translations generated by the CMM as calibration basis. The establishment of control point coordinate systems makes the solving process not complicated, and therefore can handle tens of thousands nodes on the CMM route. This will reduce the influence of random errors to a very low level. In conclusion, the proposed method is superior to previous methods.

This research was funded by State Administration of Science, Technology and Industry for National Defense of China, grant number JSJL2014206B001.

\section{References}

1. T. Várady, R.R. Martin, J. Cox, Comput. Aided Des. 29, 255-268 (1997)

2. S.G Liu, K. Peng, F.S. Huang, G.X. Zhang, P. Li, Key Eng. Mater. 295-296, 331-336 (2005)

3. Z.X. Xie, Z.H. Han, X. Gao, Chin. Opt. 6, 780-787 (2013)

4. S.G. Liu, H.L. Zhang, Y.H. Dong, S.L. Tang, Z.Z. Jiang, Meas. Sci. Rev. 13, 194-199 (2013)

5. S. Fu, L.Y. Zhang, N. Ye, W.Z. Zhang, S.L. Liu, Meas. Sci. Technol. 25, 1-8 (2014)

6. R. Tsai, IEEE J. Robot. Autom. 3, 323-344 (1987)

7. Z.Y. Zhang, IEEE Trans. Pattern Anal. Mach. Intell. 22, 1330-1334 (2000)

8. R. Zhang, S.G. Liu, S. Wang, X.X. Song, Sensors 17, 131 (2017) 